\title{
KILLING VECTOR FIELDS ON TANGENT BUNDLES WITH CHEEGER-GROMOLL METRIC
}

\author{
By \\ Mohamed Tahar Kadaoui ABbASSI and Maâti SARIH
}

\begin{abstract}
This paper deals with properties of the Cheeger-Gromoll metric $\bar{g}$ introduced in 1988 by Musso and Tricerri on the tangent bundle $T M$ associated to a given Riemannian metric $(M, g)$.

One can find here essentially the two following results:

1. A classification of Killing vector fields on $(T M, \bar{g})$

2. A generalization of a result of $\mathbf{M}$. Sekizawa concerning the non rigidity of the Cheeger-Gromoll metric.
\end{abstract}

\section{Introduction}

Let $(M, g)$ be a Riemannian manifold and $T M$ its tangent bundle with the natural projection $\pi: T M \rightarrow M$.

Although the Sasaki metric $T g$ on $T M$ [9] is a "naturally defined" Riemannian metric, it is "extremely rigid" [8]. O. Kowalski [6] has shown that it is never locally symmetric unless the base metric is locally Euclidean. E. Musso and F. Tricerri [8] have generalized this fact: they have shown that it has constant scalar curvature if and only if the base metric is flat. To surmount this hindrance E. Musso and F. Tricerri have suggested the Cheeger-Gromoll metric $\bar{g}$ on $T M$ [3] as an alternative. Indeed, M. Sekizawa [10] has shown that its scalar curvature is never constant if the original metric on the base manifold has constant curvature. The authors have also studied some other properties of this metric [1], many of which will be used here.

Throughout this work, $T M$ will be endowed with the Cheeger-Gromoll metric $\bar{g}$. We give, firstly, a theorem on general forms of all Killing vector fields on

Mathematics subject classification: 53B20, 53C07, 53C20, 53C24.

Key words and phrases: Riemannian manifolds, tangent bundle, Sasaki metric, Cheeger-Gromoll metric, classification, Killing vector fields, curvatures, rigidity of metrics.

Received November 15, 2001.

Revised May 15, 2002. 
$(T M, \bar{g})$ (Theorem 3.6). If some assumptions are imposed, then the forms of Killing vector fields become simpler ones (Theorems 4.1, 4.2 and 4.3); some ideas of these results are inspired from similar ones for the case of the Sasaki metric [12]. In $\S 5$, we give a generalization of a result of M. Sekizawa [10] concerning the non rigidity of the Cheeger-Gromoll metric on the tangent bundle (Theorem 5.7).

In this paper, manifolds are assumed to be connected and smooth and the so-called "Einstein's summation" will be used everywhere.

The authors would like to thank Professor M. Sekizawa for his helpful comments on this work.

\section{Preliminaries}

A local coordinate neighborhood $\left\{\left(U ; x^{i}, i=1, \ldots, n\right)\right\}$ in $M$ induces on $T M$ a local coordinate neighborhood $\left.\left\{\pi^{-1}(U) ; x^{i}, v^{i}, i=1, \ldots, n\right)\right\}$. Let $X=\left(X^{i}\right)$ be a vector field on $M$. Then the complete lift $X^{c}$, the vertical lift $X^{v}$ and the horizontal lift $X^{h}$ of $X$ are given, with respect to the induced coordinates, by: $X^{c}=\left(X^{i}, v^{j}\left(\partial X^{i} / \partial X^{j}\right)\right), X^{v}=\left(0, X^{i}\right)$ and $X^{h}=\left(X^{i},-\Gamma_{j k}^{i} v^{j} X^{k}\right)$ respectively, where $\left(\Gamma_{j k}^{i}\right)$ denote the Christoffel's symbols of $g$. (cf. [9], [15])

On the other hand, to any $(1,1)$-tensor field $P$ on $M$, one can associate two vector fields $l P$ and ${ }^{*} P$ defined on $T M$ by:

$$
\imath P=\left(0, P_{j}^{i} v^{j}\right) \text { and }{ }^{*} P=\left(P_{j}^{i} v^{j},-\Gamma_{l k}^{i} P_{j}^{l} v^{j} v^{k}\right) .
$$

Then $l P$ is a vertical vector field and ${ }^{*} P$ is a horizontal one, and we have $X^{c}=X^{h}+\imath(\nabla X)$, where $\nabla X=\left(\nabla_{j} X^{i}\right)$ and $\nabla$ denotes the covariant differentiation with respect to the Levi-Civita connection of $g$.

In this paper, we need new lifts of vector fields on $M$. For any vector field $X$ on $M$, let $X_{A}$ and $X_{B}$ be vector fields on $T M$ defined at any point $(x, u) \in T M$ by

$$
\begin{gathered}
X_{A}(x, u)=\left(1-r^{2}\right) X^{v}(x, u)+g_{x}(u, X(x)) U(x, u), \\
X_{B}(x, u)=\left(1-r^{2}\right) X^{v}(x, u)+g_{x}(u, X(x)) U(x, u)+{ }^{*}(C(X))(x, u),
\end{gathered}
$$

where $r^{2}=g_{x}(u, u), U$ is the canonical vertical vector field defined, locally, by $U=\left(0, v^{i}\right)$, i.e., $U(x, u)=\left(0, u^{i}\right)$, and $C(X)$ is $(1,1)$-tensor field on $M$ defined by $g(C(X) Y, Z)=-g\left(Y, \nabla_{Z} X\right)$ for all vector fields $Y$ and $Z$ on $M$. The components of $C(X)$ with respect to the local coordinates are $\left(-g^{i k} g_{j l} \nabla_{k} X^{l}\right)$. Clearly the lifts $X_{A}$ and $X_{B}$ are smooth vector fields on $T M$.

Remark that $X_{B}$ is a vertical field; it is the vertical part of $X_{A}$.

The Cheeger-Gromoll metric $\bar{g}$ on $T M$ has components [1]: 


$$
\left\{\begin{array}{l}
\bar{g}_{i j}=g_{i j}+v^{\lambda} v^{\tau} \Gamma_{i \lambda}^{k} \Gamma_{j \tau}^{l} G_{k l} \\
\bar{g}_{i n+j}=v^{\tau} \Gamma_{i \tau}^{k} G_{k j} \\
\bar{g}_{n+i n+j}=G_{i j}
\end{array} \quad i, j=1, \ldots, n,\right.
$$

where $G_{i j}$ is the function on $\pi^{-1}(U)$ defined by:

$$
G_{i j}=1 /\left(1+r^{2}\right)\left(g_{i j}+g_{i k} g_{j l} v^{k} v^{l}\right) .
$$

\section{Classification of Killing Vector Fields on $(T M, \bar{g})$}

The general forms of Killing vector fields on $(T M, \bar{g})$ are given by

THEOREM 3.1. Let $(T M, \bar{g})$ be the tangent bundle with the Cheeger-Gromoll metric of a Riemannian manifold $(M, g)$. Let

(i) $X$ be a Killing vector field on $(M, g)$;

(ii) $P$ be a $(1,1)$-tensor field on $(M, g)$ which is

$\left(P_{1}\right)$ Parallel with respect to $g$ and

$\left(P_{2}\right)$ skew-symmetric with respect to $g$, when considered as a vector field valued linear mapping on the space of vector fields on $M$;

(iii) $Y$ be a vector field on $(M, g)$ which satisfies

$\left(Y_{1}\right)$ the second covariant derivation $\nabla^{2} Y$ of $Y$ is skew-symmetric, when considered as a vector field valued bilinear mapping on the space of vector fields on $M$;

$$
\begin{aligned}
-R(X, C(Y)(W)) V-R(X, C(Y)(V)) W \\
\quad=2\left[g\left(\nabla_{X} Y, V\right) W+g\left(\nabla_{X} Y, W\right) V-2 g(V, W) \nabla_{X} Y\right]
\end{aligned}
$$

for all vector fields $X, V$ and $W$ on $M$.

Then the vector field $Z$ on $T M$ defined by

(TK) $Z=X^{c}+\imath P+Y_{B}$ is a Killing vector field on $(T M, \bar{g})$.

Conversely, every Killing vector field on $(T M, \bar{g})$ is of the form $(T K)$.

ProOF. The proof will be devided into a series of propositions and lemmas.

We have shown in [1] (Theorem 5.11), that the vertical lift to $T M$ of a nonzero vector field on $M$ is never a Killing vector on $(T M, \bar{g})$ contrary to the Sasaki metric case where the vertical lift to $T M$ of a parallel vector field on $M$ is a Killing vector field on $(T M, T g)$ [9]. This fact for the Sasaki metric was a fundamental tool on which $\mathrm{S}$. Tanno had based to give general forms of all Killing vector fields on $(T M, T g)$ [12]. Now, we will give an analogous of Tanno's the- 
orem (Theorem A. [12]) on the classification of Killing vector fields, in the case of Cheeger-Gromoll metric.

Recall that a vector field $Z=\left(Z^{I}\right)$ is a Killing vector or an infinitesimal isometry with respect to the metric $\bar{g}=\left(\bar{g}_{I J}\right)$ if and only if $L_{Z} \bar{g}=0$, where $L_{Z}$ denotes the Lie derivative with respect to $Z$, i.e.,

$$
Z^{K} \partial_{K} \bar{g}_{I J}+\partial_{I} Z^{K} \bar{g}_{K J}+\partial_{J} Z^{K} \bar{g}_{K I}=0 ; \quad I, J=1, \ldots, 2 n .
$$

Putting $(I, J)=(i, j),(i, n+j)$ and $(n+i, n+j)$, the last equation is transformed to the system:

$$
\left\{\begin{array}{c}
\left(K_{1}\right) \quad Z^{\gamma} \partial / \partial x^{\gamma}\left(g_{i j}+v^{\lambda} v^{\tau} \Gamma_{i \lambda}^{k} \Gamma_{j \tau}^{l} G_{l k}\right)+Z^{n+\gamma} \partial / \partial v^{\gamma}\left(g_{i j}+v^{\lambda} v^{\tau} \Gamma_{i \lambda}^{k} \Gamma_{j \tau}^{l} G_{k l}\right) \\
+\partial Z^{\gamma} / \partial x^{i}\left(g_{\gamma j}+v^{\lambda} v^{\mu} \Gamma_{\gamma \lambda}^{k} \Gamma_{j \mu}^{l} G_{k l}\right)+\partial Z^{n+\gamma} / \partial x^{i} v^{\mu} \Gamma_{j \mu}^{k} G_{k \gamma} \\
+\partial Z^{\gamma} / \partial x^{j}\left(g_{\gamma i}+v^{\lambda} v^{\mu} \Gamma_{\gamma \lambda}^{k} \Gamma_{i \mu}^{l} G_{k l}\right)+\partial Z^{n+\gamma} / \partial x^{j} v^{\lambda} \Gamma_{i \lambda}^{k} G_{k \gamma}=0 \\
\left(K_{2}\right) \quad Z^{\gamma} \partial / \partial x^{\gamma}\left(v^{\lambda} \Gamma_{i \lambda}^{k} G_{k j}\right)+Z^{n+\gamma} \partial / \partial v^{\gamma}\left(v^{\lambda} \Gamma_{i \lambda}^{k} G_{k j}\right)+\partial Z^{\gamma} / \partial x^{i} v^{\lambda} \Gamma_{\gamma \lambda}^{k} G_{k j} \\
+\partial Z^{n+\gamma} / \partial x^{i} G_{\gamma j}+\partial Z^{\gamma} / \partial v^{j}\left(g_{\gamma i}+v^{\lambda} v^{\tau} \Gamma_{i \lambda}^{k} \Gamma_{\gamma \tau}^{l} G_{k l}\right) \\
+\partial Z^{n+\gamma} / \partial v^{j} v^{\lambda} \Gamma_{i \lambda}^{k} G_{k \gamma}=0 \\
\left(K_{3}\right) \quad Z^{\gamma} \partial G_{i j} / \partial x^{\gamma}+Z^{n+\gamma} \partial G_{i j} / \partial v^{\gamma}+\partial Z^{\gamma} / \partial v^{i} v^{\lambda} \Gamma_{\gamma \lambda}^{k} G_{k j}+\partial Z^{n+\gamma} / \partial v^{i} G_{\gamma j} \\
+\partial Z^{\gamma} / \partial v^{j} v^{\lambda} \Gamma_{\gamma \lambda}^{k} G_{k i}+\partial Z^{n+\gamma} / \partial v^{j} G_{\gamma i}=0
\end{array}\right.
$$

First of all, we shall study the particular cases $X^{c}, \imath P$ and $X_{B}$.

Proposition 3.2 [1]. In order that a complete lift $X^{c}$ to $T M$ of a vector field $X$ on $M$ be a Killing vector field of $(T M, \bar{g})$, it is necessary and sufficient that $X$ itself is a Killing vector field of $(M, g)$.

The following lemma is immediate from $(K)$ :

\section{LEMMA 3.3.}

1- If $P=\left(P_{j}^{i}\right)$ is a $(1,1)$-tensor field on $M$, then:

$$
L_{\imath P} \bar{g}=\left(\begin{array}{cc}
0 & G_{\gamma i} \nabla_{j} P_{r}^{\gamma} v^{r} \\
G_{\gamma j} \nabla_{i} P_{r}^{\gamma} v^{r} & \frac{1}{1+r^{2}}\left(A_{i j}+B_{i j}-C_{i j}\right)
\end{array}\right),
$$

where 


$$
\begin{aligned}
A_{i j} & =\left(g_{\gamma j} P_{i}^{\gamma}+g_{\gamma i} P_{j}^{\gamma}\right)+\left(g_{\gamma i} P_{r}^{\gamma}+g_{\gamma r} P_{i}^{\gamma}\right) v^{r} v_{j} \\
B_{i j} & =\left(g_{\gamma j} P_{r}^{\gamma}+g_{\gamma r} P_{j}^{\gamma}\right) v^{r} v_{i}, \\
C_{i j} & =\left(g_{\gamma s} P_{r}^{\gamma}+g_{\gamma r} P_{s}^{\gamma}\right) v^{r} v^{s} G_{i j} .
\end{aligned}
$$

2- If $Y$ is a vector field on $M$, then

$$
L_{Y_{B}} \bar{g}=\left(\begin{array}{cc}
-\left(\nabla_{i} \nabla_{j} Y^{k}+\nabla_{j} \nabla_{i} Y^{k}\right) v^{k} & D_{i j} \\
D_{j i} & 0
\end{array}\right),
$$

where $D_{\xi \zeta}=-g^{l k} G_{k \xi} v^{r} v^{s}\left[R_{l s \zeta \gamma} \nabla^{\gamma} Y_{r}-\nabla_{\zeta} Y^{\gamma}\left(g_{l r} g_{\gamma s}+g_{l s} g_{\gamma r}-2 g_{l \gamma} g_{r s}\right)\right], \xi, \zeta=1, \ldots, n$.

As corollaries of lemma 3.3 , we obtain

Proposition 3.4. Let $P$ be a $(1,1)$-tensor field on $(M, g)$ satisfying the conditions $\left(P_{1}\right)$ and $\left(P_{2}\right)$ in Theorem 3.1. Then ${ }_{\imath} P$ is a Killing vector field on $(T M, \bar{g})$.

Proposition 3.5. Let $Y$ be a vector field on $(M, g)$ satisfying the conditions $\left(Y_{1}\right)$ and $\left(Y_{2}\right)$ in Theorem 3.1. Then $Y_{B}$ is a Killing vector field on $(T M, \bar{g})$.

Now, we consider the 0 -section $\left(v^{i}=0\right)$ in the coordinate neighborhood $\left(\pi^{-1}(U) ; x^{i}, v^{i}, i=1, \ldots, n\right)$ in $T M$ induced from $\left(U ; x^{i}, i=1, \ldots, n\right)$ in $M$, and its neighborhood $W$. For a vector field $Z$ on $T M$, and $(x, v)=\left(x^{i}, v^{i}\right)$ in $W$, we can write, by Taylor's theorem,

$$
\begin{aligned}
Z^{i}(x, v)=Z^{i}(x, 0)+ & \partial Z / \partial v^{r}(x, 0) v^{r}+\frac{1}{2}\left(\partial / \partial v^{r}\left(\partial Z^{i} / \partial v^{s}\right)\right)(x, 0) v^{r} v^{s}+\cdots[*]_{\lambda}^{i}, \\
Z^{n+i}(x, v)= & Z^{n+i}(x, 0)+\partial Z^{n+i} / \partial v^{r}(x, 0) v^{r} \\
& +\frac{1}{2}\left(\partial / \partial v^{r}\left(\partial Z^{n+i} / \partial v^{s}\right)\right)(x, 0) v^{r} v^{s}+\cdots[*]_{\lambda}^{n+i}
\end{aligned}
$$

where $[*]_{\lambda}^{I}(I=1, \ldots, 2 n)$ is of the form: $[*]_{\lambda}^{I}=\frac{1}{\lambda !}\left(\partial^{\lambda} Z^{I} / \partial v^{i_{1}} \partial v^{i_{2}} \cdots \partial v^{i_{\lambda}}\right)$. $\left(x^{a}, \theta(x, v) v^{b}\right) v^{i_{1}} v^{i_{2}} \cdots v^{i_{\lambda}} ; 1 \leq i_{1}, \ldots, i_{\lambda} \leq n$.

The following lemma is valid.

LEMMA 3.6 [12]. In the above situation, the following

$$
\begin{aligned}
& X=\left(X^{i}(x)\right)=\left(Z^{i}(x, 0)\right), \quad Y=\left(Y^{i}(x)\right)=\left(Z^{n+i}(x, 0)\right), \\
& K=\left(K_{r}^{i}(x)\right)=\left(\partial Z^{i} / \partial v^{r}(x, 0)\right), \\
& E=\left(E_{r s}^{i}(x)\right)=\left(\partial / \partial v^{r}\left(\partial Z^{i} / \partial v^{s}\right)(x, 0)\right), \\
& P=\left(P_{r}^{i}(x)\right)=\left(\left(\partial Z^{n+i} / \partial v^{r}\right)(x, 0)-\partial Z^{i} / \partial x^{r}(x, 0)\right)
\end{aligned}
$$

are tensor fields on $M$. 
With the notations of lemma 3.6 , we can write:

$\left(T_{1}\right) Z^{i}(x, v)=X^{i}+K_{r}^{i} v^{r}+\frac{1}{2} E_{r s}^{i} v^{r} v^{s}+\cdots[*]_{\lambda}^{i}$,

$\left(T_{2}\right) Z^{n+i}(x, v)=Y^{i}+\tilde{P}_{r}^{i} v^{r}+\frac{1}{2} Q_{r s}^{i} v^{r} v^{s}+\cdots+[*]_{\lambda}^{n+i}$,

where $\tilde{P}_{r}^{i}$ and $Q_{r s}^{i}$ are given by

$$
\tilde{P}_{r}^{i}(x)=\partial Z^{n+i} / \partial v^{r}(x, 0) \text { and } Q_{r s}^{i}=\partial^{2} Z^{n+i} / \partial v^{r} \partial v^{s}(x, 0),
$$

and we have:

$\left(T_{3}\right) \partial Z^{i} / \partial v^{j}(x, v)=K_{j}^{i}+E_{r j}^{i} v^{r}+\cdots+\langle *\rangle_{\lambda-1}^{i}$,

$\left(T_{4}\right) \partial Z^{n+i} / \partial v^{j}(x, v)=\tilde{P}_{j}^{i}+Q_{r j}^{i} v^{r}+\cdots+\langle *\rangle_{\lambda-1}^{n+i}, \ldots$ etc.

When we apply Taylor's theorem to the left hand sides of $(K)$ to some order $\lambda$, the results are the same as one obtains by substituting $\left(T_{1}\right),\left(T_{2}\right),\left(T_{3}\right),\left(T_{4}\right) \ldots$ into $(K)$ up to order $\lambda-1$. Furthermore the vanishing of the right hand sides of the equations of $(K)$ implies the vanishing of each coefficient (up to order $\lambda-1$ ).

Substituting $\left(T_{1}\right)$ and $\left(T_{2}\right)$ into $\left(K_{1}\right)$ and taking the part which does not contain $v^{r}$, we have

$$
X^{\gamma} \partial g_{i j} / \partial x^{\gamma}+\partial X^{\gamma} / \partial x^{i} g_{\gamma j}+\partial X^{\gamma} / \partial x^{j} g_{\gamma i}=0
$$

Hence, $X=\left(X^{i}\right)$ is a Killing vector field on $(M, g)$. Since, by proposition $3.2, X^{c}$ is a Killing vector field on $(T M, \bar{g}), Z-X^{c}$ is also a Killing vector field. Therefore, in the following, denoting $Z-X^{c}$ by the same letter $Z$, one may assume that $X^{i}=0$ in $\left(T_{1}\right)$. Then $\left(\tilde{P}_{r}^{i}\right)=\left(P_{r}^{i}\right)$ is a tensor field on $M$ by lemma 3.6.

Putting $\left(T_{1}\right)$ and $\left(T_{2}\right)$ into $\left(K_{2}\right)$ (from now on, we omit this statement) and taking the part which does not contain $v^{r}$, we get: $Y^{\gamma} \Gamma_{i \gamma}^{k} g_{k j}+\partial Y^{\gamma} / \partial x^{i} g_{\gamma j}+$ $K_{j}^{\gamma} g_{\gamma i}=0, \quad$ that is

$\left(D_{1}\right) K_{j}^{\gamma} g_{i \gamma}=-g_{\gamma j} \nabla_{i} Y^{\gamma}=-\nabla_{i} Y_{j}$, i.e.,

$\left(D_{1}^{\prime}\right) K_{j}^{i}=-\nabla^{i} Y_{j}$.

Taking the coefficient of $v^{s}$ in $\left(K_{1}\right)$, we get

$$
\begin{aligned}
& K_{s}^{\gamma} \partial g_{i j} / \partial x^{\gamma}+Y^{\gamma}\left(\Gamma_{i s}^{k} \Gamma_{j \gamma}^{l} g_{l k}+\Gamma_{i \gamma}^{k} \Gamma_{j s}^{l} g_{l k}\right)+\partial K_{s}^{\gamma} / \partial x^{i} g_{\gamma j} \\
& \quad+\partial Y^{\gamma} / \partial x^{i} \Gamma_{j s}^{k} g_{k \gamma}+\partial K_{s}^{\gamma} / \partial x_{j} g_{\gamma i}+\partial Y^{\gamma} / \partial x^{j} \Gamma_{i s}^{k} g_{k \gamma}=0 .
\end{aligned}
$$

Using the equality $\partial g_{i j} / \partial x^{\gamma}=\Gamma_{\gamma i}^{t} g_{t j}+\Gamma_{\gamma j}^{t} g_{t i}$ and $\left(D_{1}\right)$, we see that the above equation can be simplified to

$\left(D_{2}\right) \nabla_{i} \nabla_{j} Y_{s}+\nabla_{j} \nabla_{i} Y_{s}=0$

Taking the part which does not contain $v^{r}$, in $\left(K_{3}\right)$, we get

$\left(D_{3}\right) P_{i}^{\gamma} g_{\gamma j}+P_{j}^{\gamma} g_{\gamma i}=0$.

Taking the coefficient of $v^{s}$ in $\left(K_{2}\right)$, we get

$$
P_{s}^{\gamma} \Gamma_{i \gamma}^{k} g_{k j}+\partial P_{s}^{\gamma} / \partial x^{i} g_{\gamma j}+E_{s j}^{\gamma} g_{\gamma i}+P_{j}^{\gamma} \Gamma_{i s}^{k} g_{k \gamma}=0
$$


or equivalently

$$
g_{i \gamma} E_{j s}^{\gamma}+\nabla_{i} P_{s}^{\gamma} g_{j \gamma}=0, \quad \text { i.e. } g_{i \gamma} E_{j s}^{\gamma}+\nabla_{i} P_{s j}=0
$$

where $\boldsymbol{P}_{s j}=\boldsymbol{P}_{s}^{\gamma} g_{\gamma j}$.

Since $E_{j s}^{\gamma}$ is symmetric in $j$ and $s$, and $\left(\nabla_{i} P_{s}^{\gamma}\right) g_{\gamma j}=\nabla_{i} P_{s j}$ is skew-symmetric in $j$ and $s$ by $\left(D_{3}\right)$, we have

$\left(D_{4}\right) E_{j s}^{\gamma}=0$ and $\nabla_{i} P_{s}^{\gamma}=0$.

Taking the coefficient of $v^{s}$ in $\left(K_{3}\right)$, we get

$$
\begin{gathered}
K_{s}^{\gamma} \partial g_{i j} / \partial x^{\gamma}+Y^{\gamma}\left(g_{i s} g_{j \gamma}+g_{i \gamma} g_{j s}-2 g_{\gamma s} g_{i j}\right)+K_{i}^{\gamma} \Gamma_{\gamma s}^{k} g_{k j} \\
+Q_{s i}^{\gamma} g_{\gamma j}+K_{j}^{\gamma} \Gamma_{\gamma s}^{k} g_{k i}+Q_{s j}^{\gamma} g_{\gamma i}=0 .
\end{gathered}
$$

We put $Q_{r s}^{i}=-\left(\Gamma_{k r}^{i} K_{s}^{k}+\Gamma_{k s}^{i} K_{r}^{k}\right)-2 Y^{i} g_{r s}+\left(Y^{k} g_{k r} \delta_{s}^{i}+Y^{k} g_{k s} \delta_{r}^{i}\right)+T_{r s}^{i}$.

By simple calculation using $\left(D_{5}\right)$, we can verify that

$$
T_{i s}^{r} g_{r j}+T_{j s}^{r} g_{r i}=0 .
$$

If we put $T_{i s j}=T_{i s}^{r} g_{r j}$, then $T_{i s j}$ is symmetric in $i$ and $s$, and skew-symmetric in $i$ and $j$. Hence $T_{i s j}=0$. That is

$\left(D_{6}\right) Q_{s r}^{i}=-\left(\Gamma_{k r}^{i} K_{s}^{k}+\Gamma_{k s}^{i} K_{r}^{k}\right)-2 Y^{i} g_{r s}+\left(Y^{k} g_{k r} \delta_{s}^{i}+Y^{k} g_{k s} \delta_{r}^{i}\right)$.

Taking the coefficient of $v^{s} v^{t}$ in $\left(K_{2}\right)$, we get by virtue of $\left(D_{6}\right)$

$$
\begin{aligned}
& \left(K_{s}^{\gamma} R_{i t \gamma}^{k}+K_{t}^{\gamma} R_{i s \gamma}^{t}\right) g_{k j}+2 \nabla_{i} Y^{\gamma}\left(g_{\gamma s} g_{j t}+g_{\gamma t} g_{j s}-2 g_{\gamma j} g_{s t}\right) \\
& \quad+\partial / \partial v^{s}\left(\partial / \partial v^{t}\left(\partial Z^{\gamma} / \partial v^{j}\right)\right)(x, 0) g_{\gamma i}=0
\end{aligned}
$$

Taking the skew-symmetric part in $s$ and $j$ of $\left(D_{7}\right)$, we get $\left(D_{8}\right) 2 R_{j s i \gamma} K_{t}^{\gamma}+R_{j t i \gamma} K_{s}^{\gamma}-R_{s t i \gamma} K_{j}^{\gamma}+2 \nabla_{i} Y^{\gamma}\left(3 g_{\gamma s} g_{j t}-3 g_{\gamma j} g_{s t}\right)=0$.

Taking the symmetric part in $s$ and $t$ of $\left(D_{8}\right)$, we get

$\left(D_{9}\right) R_{j s i \gamma} K_{t}^{\gamma}+R_{j t i \gamma} K_{s}^{\gamma}=-2 \nabla_{i} Y^{\gamma}\left(g_{\gamma s} g_{j t}+g_{\gamma t} g_{j s}-2 g_{\gamma j} g_{s t}\right)$.

Now, by $\left(D_{3}\right)$ and $\left(D_{4}\right)$, we see that $\imath P$ is a Killing vector on $(T M, \bar{g})$ by Proposition 3.4. By $\left(D_{1}\right),\left(D_{2}\right),\left(D_{9}\right)$ and Proposition 3.5, $Y_{B}$ is a Killing vector field on $(T M, \bar{g})$.

We put $[Z]=Z-\imath P-Y_{B}$ which is a Killing vector field on $(T M, \bar{g})$. Using the preceding arguments, we see that $\left([Z]^{A}\right)(x, 0)=\left(\partial[Z]^{A} / \partial x^{B}\right)(x, 0)=0$, for $A=i, n+i$ and $B=j, n+j$ at a point $p=(x, 0)$.

Since a Killing vector field $[Z]$ (or more generally an infinitesimal affine transformation) is determined by the values of its components and their first partial derivatives at a point (cf. [5, p. 232]), we have $[Z]=0$ on $T M$.

Finally, propositions 3.2, 3.4 and 3.5 and the facts that have already been shown complete the proof of Theorem 3.1. 


\section{Classification of Killing Vector Fields on $(T M, \bar{g})$ in Particular Cases}

Let $(M, g)$ be a compact oriented Riemannian manifold with volume element $d v$. We can define the global scalar product $\langle\alpha, \beta\rangle_{g}$ of two $p$-forms $\alpha$ and $\beta$ as follows: $\langle\alpha, \beta\rangle_{g}=\int_{M} g(\alpha, \beta) d v$, where $g(\alpha, \beta)$ denotes the scalar product of $\alpha$ and $\beta$, with respect to $g$, locally defined by $g(\alpha, \beta)=\alpha_{i_{1} \ldots i_{p}} \beta^{i_{1} \cdots i_{p}}$.

The norm associated to the global product $\langle,\rangle_{g}$ is denoted by \|\|$_{\langle,\rangle_{g}}$.

THEOREM 4.1. In theorem 3.1, if $(M, g)$ is compact, then $Y$ satisfying $\left(Y_{1}\right)$ and $\left(Y_{2}\right)$ is parallel. Hence $(T K)$ is $Z=X^{c}+\imath P+Y_{A}$.

Proof. By considering the double covering manifold if necessary, we can assume that $M$ is orientable. Let $Y$ be a vector field on $M$ satisfying $\left(Y_{1}\right)$. We can write, using $\left(Y_{1}\right), \nabla_{i} \nabla^{i} Y_{r}=\nabla_{i}\left(g^{i j} \nabla_{j} Y_{r}\right)=g^{i j} \nabla_{i} \nabla_{j} Y_{r}=0$. In this case, we have

( $\alpha) \nabla_{i}\left(Y_{r} \nabla^{i} y^{r}\right)=\nabla_{i} Y_{r} \nabla^{i} Y^{r}+Y_{r} \nabla_{i} \nabla^{i} Y^{r}=\nabla_{i} Y_{r} \nabla^{i} Y^{r}$.

If we denote by $Y^{*}$ the 1-form on $M$ obtained by contraction of $Y$ with respect to $g$, then the quantity $Y_{r} \nabla^{i} Y^{r}$ is the local expression of the vector field $V$ obtained by contraction of the $(2,1)$-tensor field $Y^{*} \otimes \nabla_{*} Y ; \nabla_{*} Y$ being defined locally by, $\left(\nabla_{*} Y\right)^{i j}=\nabla^{i} Y^{j}$.

On the other hand, the quantity $\nabla_{i} Y_{r} \nabla^{i} Y^{r}$ is nothing but $g\left(\nabla Y^{*}, \nabla Y^{*}\right)$. The equality $(\alpha)$ is then transformed to $\nabla_{i} V^{i}=\left(\nabla Y^{*}\right)_{i r}\left(\nabla Y^{*}\right)^{i r}$, that is

( $\beta) \operatorname{div} V=g\left(\nabla Y^{*}, \nabla Y^{*}\right)$.

Integrating both sides of the last equation $(\beta)$, we get

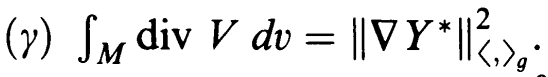

Applying Green's theorem $\left(\int_{M} \operatorname{div} V d v=0\right.$ [5], I.p. 281), it follows from $(\gamma)$ that $\left\|\nabla Y^{*}\right\|_{\langle,\rangle_{g}}^{2}=0$.

Thus $\nabla Y^{*}=0$ i.e. $\nabla Y=0$. Therefore $Y_{B}=Y_{A}$, which gives the result.

THEOREM 4.2. In theorem 3.1, if $(M, g)$ satisfies one of the following three conditions:

(i) $M$ is compact and orientable with vanishing first Betti number $b_{1}(M)$;

(ii) $M$ is compact and orientable with strictly positive Ricci Curvature;

(iii) $(M, g)$ is an Einstein space with non-zero scalar curvature different from $2 n(1-n)$, then $Y$ satisfying $\left(Y_{1}\right)^{\prime}$ and $\left(Y_{2}\right)$ vanishes, in which case $(T K)$ becomes $Z=X^{c}+\imath P$.

Proof. (i) $M$ being compact, $Y$ satisfying $\left(Y_{1}\right)$ and $\left(Y_{2}\right)$ is parallel, by theorem 4.1. Applying Hodge's theorem ([13]), we have $Y=0$ by virtue of $b_{1}(M)=0$. 
(ii) According to [2, theorem 1.76], $b_{1}(M)=0$. So, we can apply (i).

(iii) contracting $\left(Y_{2}\right)$ with respect to $i$ and $s$, we obtain

$$
R_{s \gamma} \nabla^{\gamma} Y_{t}+R_{t \gamma} \nabla^{\gamma} Y_{s}=2\left(\nabla_{t} Y_{s}+\nabla_{s} Y_{t}-2 \nabla^{r} Y_{r} g_{s t}\right),
$$

where $\left(R_{i j}\right)$ denotes the Ricci curvature tensor.

By assumption, $(M, g)$ satisfies: $R_{s \gamma}=S / n g_{s \gamma}$, for non-zero scalar curvature $S$. Hence, we have

$$
(S / n-2)\left(\nabla_{s} Y_{t}+\nabla_{t} Y_{s}\right)=-4 \nabla^{r} Y_{r} g_{s t} .
$$

Multiplying by $g^{s t}$ both sides of the last equation and summing over $s$ and $t$, we get $\left(2 n^{2}-2 n+S\right) \nabla^{s} Y_{s}=0$.

Since $S \neq 2 n(1-n)$, we have $\nabla^{s} Y_{s}=0$, which means that $\nabla_{r} Y^{r}=0$. Now, using $\left(Y_{1}\right)$ and the Ricci identity,

$$
\nabla_{i} \nabla_{j} Y^{r}-\nabla_{j} \nabla_{i} Y^{r}=R_{t j i}^{r} Y^{t},
$$

we get

$$
\nabla_{i} \nabla_{j} Y^{r}=\frac{1}{2} R_{t j i}^{r} Y^{t} .
$$

Contracting with respect to $r$ and $j$, and applying $\nabla_{r} Y^{r}=0$, we get $R_{t i} Y^{t}=0$, that is $S / n g_{t i} Y^{t}=S / n Y_{i}=0$. Therefore $Y=0$, since $S \neq 0$.

THEOREM 4.3. Let $(T M, \bar{g})$ be the tangent bundle with the Cheeger-Gromoll metric $\bar{g}$ of a Riemannian manifold $(M, g)$. If $M$ is compact and orientable, and if the first and the second Betti numbers vanish, then the Lie algebra of Killing vector fields on $(M, g)$ and the Lie algebra of Killing vector fields on $(T M, \bar{g})$ are isomorphic, via the correspondence $X \rightarrow X^{c}$.

Proof. Applying theorem 4.2(i), it follows from $b_{1}(M)=0$ that $Y=0$. Furthermore, if the $(1,1)$-tensor field $P$ is parallel then, by $b_{2}(M)=0$ and Hodge's theorem, we have $P=0$. Therefore, every Killing vector field on $(T M, \bar{g})$ is of the form $X^{c}$ for some Killing vector field $X$ on $(M, g)$. On the other hand, for any vector fields $X$ and $X^{\prime}$ on $M$, it is known that $\left[X, X^{\prime}\right]^{c}=\left[X^{c}, X^{\prime c}\right]$.

REMARK 4.4. Let $(M, g)$ be a compact orientable Riemannian manifold whose first and second Betti numbers vanish. The isomorphism stated in theorem 4.3 is also true in the case of the Sasaki metric [12, Theorem D]. Generally, one can consider the following interesting questions: Consider on $T M$ a Riemannian metric $G$ so that: 
(i) The projection $\pi:(T M, G) \rightarrow(M, g)$ is a Riemannian submersion;

(ii) vertical and horizontal distributions are orthogonal,

1. Is the complete lift of any Killing vector field on $(M, g)$ a Killing vector field on $(T M, G)$ ?

2. If yes, are the Lie algebras of Killing vector fields of $(M, g)$ and $(T M, G)$ isomorphic with the correspondence $X \rightarrow X^{c}$ ?

\section{The Non-Rigidity of the Cheeger-Gromoll Metric}

Concerning the rigidity of the Cheeger-Gromoll metric, M. Sekizawa had shown in [10, p. 417], the following result

THEOREM 5.1 [10]. The scalar curvature of the tangent bundle $(T M, \bar{g})$ with the Cheeger-Gromoll metric $\bar{g}$ of a Riemannian manifold of constant sectional curvature $(M, g)$ is never constant. In particular, $(T M, \bar{g})$ is never a space of constant sectional curvature if $(M, g)$ is of constant sectional curvature.

Our aim in this section is to generalize the particular situation of the previous theorem. We begin with a classical result concerning the characterization of spaces of constant sectional curvature in terms of dimension of the Lie algebra of Killing vector fields. Such a result is well known in literature (See for example [5, I. p. 238]).

THEOREM $5.2[5, \mathrm{I}]$. The Lie algebra $i(M)$ of infinitesimal isometries of a connected Riemannian manifold $M$ is of dimension at most $n(n+1) / 2$, where $n=\operatorname{dim} M$. If $\operatorname{dim} i(M)=n(n+1) / 2$, then $M$ is a space of constant curvature.

Now, we consider three subsets of $i(T M)$

$$
i_{c}(T M)=\left\{X^{c} \mid X \in i(M)\right\}
$$

$i_{l}(T M)=\left\{l P \mid P\right.$ is a $(1,1)$-tensor field on $M$ satisfying $\left(P_{1}\right)$ and $\left.\left(P_{2}\right)\right\}$ and $i_{B}(T M)=\left\{Y_{B} \mid Y\right.$ is a vector field on $M$ satisfying $\left(Y_{1}\right)$ and $\left.\left(Y_{2}\right)\right\}$.

\section{Proposition 5.3.}

1. $i_{c}(T M), i_{l}(T M)$ and $i_{B}(T M)$ are $\mathbf{R}$-vector subspaces of $i(T M)$. Furthermore, $\operatorname{dim} i_{c}(T M) \leq(1 / 2) n(n+1), \operatorname{dim} i_{l}(T M) \leq(1 / 2) n(n-1)$, and $\operatorname{dim} i_{B}(T M)$ $\leq n^{2}+n$.

2. $i(T M)=i_{c}(T M) \oplus i_{l}(T M) \oplus i_{B}(T M)$. 
3. $\operatorname{dim} i(T M)$ is maximal if and only if the dimensions of $i_{c}(T M), i_{l}(T M)$ and $i_{B}(T M)$ are maximal equal, respectively, to $(1 / 2) n(n+1),(1 / 2) n(n-1)$ and $n^{2}+n$.

Proof. 1. Using Theorem 5.2, it is clear that $\operatorname{dim} i_{c}(T M) \leq(1 / 2) n(n+1)$, since $\operatorname{dim} i(M) \leq(1 / 2) n(n+1)$. On the other hand, since elements of $i_{l}(T M)$ raise from skew-symmetric parallel $(1,1)$-tensor fields, we see that $\operatorname{dim} i_{l}(T M) \leq$ $(1 / 2) n(n-1)$. Finally, condition $\left(Y_{1}\right)$ on elements of $i_{B}(T M)$ let us assert that $\operatorname{dim} i_{B}(T M) \leq n^{2}+n$. 2. Follows immediately from Theorem 3.1. 3. Follows from 1 and 2 [Remark that $(1 / 2) n(n+1)+(1 / 2) n(n-1)+n^{2}+n=n(2 n+1)$, which is the maximal dimension of $i(T M)]$.

Now, if $(M, g)$ is of constant sectional curvature which is assumed to be different from -2 and 0 , then we can easily verify that $(M, g)$ is an Einstein space with scalar curvature different from $2 n(1-n)$ and 0 ; hence $i_{B}(T M)$ is reduced to the 0 -section of $T M$ by virtue of Theorem 4.2.

Applying 3 of Proposition 5.3, we have $\operatorname{dim} i(T M)<n(2 n+1)$. This implies, by Theorem 5.2, that $(T M, \bar{g})$ is of non constant sectional curvature.

REMARK 5.4. The additional condition on the constant sectional curvature of $(M, g)$ is not a geometrical constraint. It is nothing but a technical condition which comes from the analysis of equation $\left(Y_{1}\right)$ in Theorem 4.2. In fact, by Theorem 5.1, we can say that $(T M, \bar{g})$ is never a space of constant sectional curvature if $(M, g)$ is a space of constant sectional curvature.

On the other hand, if we suppose that $(M, g)$ is a space of non constant sectional curvature, then by Theorem 5.2, $\operatorname{dim} i(M)<(1 / 2) n(n+1)$ and so $\operatorname{dim} i_{c}(T M)<(1 / 2) n(n+1)$. Therefore, $\operatorname{dim} i(T M)<n(2 n+1)$, by 3 of Proposition 5.3.

Applying again Theorem 5.2 to the case of $T M$, we see that $(T M, \bar{g})$ is a space of non constant sectional curvature. We have then proved the following

THEOREM 5.5. The sectional curvature of the tangent bundle $(T M, \bar{g})$ with the Cheeger-Gromoll metric $\bar{g}$ of a Riemannian manifold $(M, g)$ is never constant.

REMARK 5.6. Using the same arguments as in the proof of [12, Theorem E], one can state a similar result for the Cheeger-Gromoll metric. i.e., $(M, g)$ is of constant curvature $k>1$ if and only if the restriction of the geodesic flow vector field to $T_{r} M(r=1 / \sqrt{k}-1)$, is a Killing vector field with respect to the induced metric of $(T M, \bar{g})$. 


\title{
References
}

[1] Abbassi, K. M. T. and Sarih, M. On the differential geometry of the tangent and the tangent sphere bundles with Cheeger-Gromoll metric. Preprint.

[ 2 ] Aubin, T. Nonlinear Analysis on manifolds. Monge-Ampere Equations. Grundlehren der Math. Vol. 252, New York, Heidelberg, Berlin: Springer-Verlag, 1982.

[ 3 ] Cheeger, J. and Gromoll, D. On the structure of complete manifolds of non negative curvature, Ann. of Math. 96 (1972), 413-443.

[4] Dombrowski, P. On the geometry of the tangent bundle, J. Reine Angew. Math. 210 (1962), 73-82.

[5] Kobayashi, S. and Nomizu, K. Foundations of differential geometry, Intresci-pub. New York (1963 and 1967) I and II.

[6] Kowalski, O. Curvature of the induced Riemannian metric of the tangent bundle of Riemannian manifoldd, J. Reine Angew. Math., 250 (1971), 124-129.

[ 7 ] Kowalski, O. and Sekizawa, M. Natural transformations of Riemannian metrics on manifolds to metrics on tangent bundles-a classification Bull. Tokyo Gakugei Univ. (4). 40 (1988), $1-29$.

[ 8 ] Musso, E. and Tricerri, F. Riemannian metrics on tangent bundles, Ann. Math. Pura Appl. (4), 150 (1988), 1-20.

[9] Sasaki, S. On the differential geometry of tangent bundles of Riemannian manifolds, Tohôku Math. J. (I, 10 (1958) 338-354; II, 14 (1962) 146-155).

[10] Sekizawa, M. Curvatures of tangent bundles with Cheeger-Gromoll metric, Tokyo J. Math., vol. 14, n 2 (1991), 407-417.

[11] Tanno, S. Orthonormal frames on 3-dimensional Riemannian manifolds, J. Differential geometry 11 (1976), 467-474.

[12] Tanno, S. Killing vectors and geodesic flow vectors on tangent bundles, J. Reine Angew. Math. 282 (1976), 162-171.

[13] Willmore, T. J. An introduction to differential geometry, Oxford Univ. Press, 1959.

[14] Yano, K. and Ishihara, S. Tangent and cotangent bundles, differential geometry, Marcel Dekker Inc. New York, 1973.

[15] Yano, K. and Kobayashi, S. Prolongations of tensor fields and connections to tangent bundles, J. Math. Soc. Japan (I, II, vol. 18, n 2, 3 (1966), III, vol. 19 (1967)).

\author{
Mohamed Tahar Kadaoui Abbassi \\ Département des Mathématiques \\ Faculté des sciences Dhar El Mahraz \\ Université Sidi Mohamed Ben Abdallah \\ B.P. 1796, Fes-Atlas \\ Fes, Morocco \\ e-mail: mtk_abbassi@Yahoo.fr \\ Maâti Sarih \\ Département des Mathématiques et Informatique \\ Faculté des sciences et techniques de Settat \\ Université Hassan $1^{\text {er }}$ \\ B.P. 577, 26000 Morocco
}

